\title{
Correction to: No difference in outcomes and gait analysis between mechanical and kinematic knee alignment methods using robotic total knee arthroplasty
}

\author{
Je-Hyoung Yeo ${ }^{1} \cdot$ Jong-Keun Seon $^{1} \cdot$ Dong-Hyun Lee ${ }^{1}$ - Eun-Kyoo Song ${ }^{1}$ \\ Published online: 28 May 2019 \\ ๑ European Society of Sports Traumatology, Knee Surgery, Arthroscopy (ESSKA) 2019
}

\section{Correction to: \\ Knee Surgery, Sports Traumatology, Arthroscopy (2019) 27:1142-1147 \\ https://doi.org/10.1007/s00167-018-5133-x}

The authors wish to acknowledge a confusion in alignment definition in the published paper. Instead of kinematic alignment it should read anatomical alignment. The title needs to be updated to "No difference in outcomes and gait analysis between mechanical and anatomical knee alignment methods using robotic total knee arthroplasty". Furthermore, within the text kinematic alignment needs to be replaced by anatomical alignment.
Publisher's Note Springer Nature remains neutral with regard to jurisdictional claims in published maps and institutional affiliations.

The original article can be found online at https://doi.org/10.1007/ s00167-018-5133-x.

Jong-Keun Seon

seonbell@chonnam.ac.kr

1 Center for Joint Disease, Chonnam National University Bitgoeul Hospital, 80, Deoknamgil Nam-gu, Gwangju, South Korea 